\title{
Impacto del nivel de producción, estación de parto y el tipo de servicio sobre la tasa de preñez acumulada a 100 días en vacas lecheras en la Argentina
}

Piccardi, M; A. Capitaine Funes, G.A. Bó y M. Balzarini

\begin{abstract}
RESUMEN
El propósito de este estudio fue estimar, a través de una regresión logística múltiple, la contribución relativa de factores que afectan la probabilidad de preñez a los 100 días en lactancia en rodeos lecheros argentinos. Los factores analizados fueron: nivel productivo, estación de parto, número de lactancia, tipo de servicio utilizado inseminación artificial (IA), natural o combinación de ambas y la interacción del nivel productivo y el tipo de servicio. Los 291 tambos (162.116 lactancias) son usuarios del software Dairy Comp305, del cual se obtiene la producción estimada que se espera alcance la vaca adulta (305 equivalente maduro). Todos los factores resultaron estadísticamente significativos. Las vacas con bajo nivel productivo tuvieron mayores chances de preñarse que vacas con niveles productivos mayores. Las vacas con parición en estaciones más frías tuvieron mayores chances de preñarse a los 100 días. La relación resultó cuadrática entre la probabilidad de preñarse a los 100 días y el número de lactancia. Los bovinos que recibieron servicio natural presentaron mayor tasa de preñez acumulada; sin embargo, la IA estuvo asociada a niveles productivos mayores. Los resultados sugieren la necesidad de diseños alternativos y evaluación del manejo reproductivo según niveles productivos específicos, como herramienta para incrementar la eficiencia reproductiva global.
\end{abstract}

Palabras clave: controles lecheros, 305EM, indicadores reproductivos, inseminación artificial

Piccardi, M; A. Capitaine Funes, G.A. Bó and M. Balzarini, 2011. Impact of the production level, calving season and type of service on the 100-day cumulative pregnancy rate of dairy cows in Argentina. Agriscientia XXVIII (2): 127-135

\section{SUMMARY}

The aim of this study was to estimate using a multiple logistic regression the relative contribution of factors affecting the probability of getting pregnant at the first 100 
days of milk production in Argentine dairy herds. The factors considered were milk production level, lactation number, calving season, type of service used, artificial insemination (AI), natural breeding, or combined, and the interaction between type of service and level of production. The 291 Dairy farms (162.116 lactations) are users of the software Dairy Comp 305 herd-management. This software has a production estimator called 305 mature equivalent to standardize records from different lactation numbers. The analyzed variables in the model were statistically significant. Cows of lower milk production have more chances to get pregnant than those with higher milk production. Cows that calves in colder seasons have more probabilities to get pregnant than those calves in hottest seasons. The relation was quadratic between the probability of getting pregnant at 100 days in milk and the number of lactation. The cumulative pregnancy rate at 100 days was greater with natural service, compared to Al but, the Al was related with greater levels of milk production. Therefore, the results suggest that new reproductive management designs specifically developed for given productive levels could increase the overall reproductive efficiency.

Key words: milk analysis, 305ME, reproductive indicators, artificial insemination.

M. Piccardi y M. Balzarini (CONICET). Cátedra de Estadística y Biometría, Facultad de Ciencias Agropecuarias, Universidad Nacional de Córdoba. C.C. 509, 5000 Córdoba, Argentina. A. Capitaine Funes. DAIRYTECH S.R.L. G.A. Bó. IRAC e Instituto de Ciencias Básicas, Carrera de Medicina Veterinaria, Universidad Nacional de Villa Maria, Villa del Rosario, Córdoba, Argentina. Correspondencia a: monicapiccardi@gmail.com

\section{INTRODUCCIÓN}

Los rodeos lecheros demandan prácticas de manejo reproductivo eficaces para mejorar la rentabilidad y sustentabilidad de los establecimientos. Aunque los sistemas de manejo de los rodeos comerciales varían ampliamente, el objetivo reproductivo principal es preñar a las vacas lecheras lo más rápido posible después del parto (Ferguson \& Galligan, 1993). Los indicadores más utilizados para reflejar el comportamiento reproductivo de rodeos lecheros han cambiado en los últimos años. Indicadores clásicos como el intervalo parto-parto o el intervalo parto-concepción, que equivale a los días abiertos ("days open"), no se reconocen actualmente como suficientes.

En las cuencas lecheras argentinas un indicador frecuentemente utilizado para diagnosticar el comportamiento reproductivo global de un establecimiento lechero es la tasa de preñez (TP). Ésta refleja en forma objetiva la velocidad con que se preñan las vacas (Capitaine Funes, 2002). La tasa de preñez se mide cada 21 días (un ciclo estral) y representa la proporción de vacas elegibles que se preñan en un ciclo. Cuando se habla de tasa de preñez anual se hace referencia al promedio ponderado de los 17 o 18 ciclos que tiene un año (Capitaine Funes, 2005). La TP es función de la tasa de detección de celos (TDC) y de la tasa de concepción (TC). La TDC es la proporción de vacas que se detectan en celo en un ciclo estral (21 días), mientras que la TC es la proporción de los servicios dados que originan preñeces. La TP acumulada es la proporción de vacas preñadas a un momento dado y es función de la TP. La TP acumulada a 100 días en lactancia indica la proporción de vacas que se preñan antes de los 100 días. Si se considera una espera voluntaria de 40 días, es la proporción acumulada de preñeces que se logran en tres ciclos estrales (60 días en servicio) (Capitaine Funes et al., 2004). Numerosos factores pueden afectar la TP, y la contribución relativa de éstos puede variar tanto temporal como geográficamente debido a cambios en el manejo del rodeo.

Las tres provincias que aportan el $75 \%$ de la producción nacional de leche son Córdoba (2.732.435.268 litros), Santa Fe (2.703.514.917 litros) y Buenos Aires (2.169.674.135 litros), que acumulan un total de 7.605.624.320 litros de la producción anual de la Argentina (Indicadores Lácteos, 2009). Existe gran diversidad entre los rendimientos según la provincia que se conside- 
re y también entre zonas de una misma provincia. Se observa además una gran variabilidad entre los animales; así se encuentran vacas de alta producción con más de 35 litros/día y a la vez vacas que no llegan a los 15 litros/día. Por otra parte, existen trabajos que han demostrado que la disminución en la fertilidad (celos más cortos y menos intensos) se encuentra asociada al aumento de la producción de leche (Dematawewa \& Berger, 1998; Lucy, 2001; Price \& Harris, 2004). La fertilidad decrece por una menor concentración plasmática de estrógenos debida a la metabolización en el hígado de esta hormona (Lopez et al., 2004). A su vez, la TC muestra una importante variación estacional, y la mayor concentración se da en los estaciones más frías (Lucy, 2001; Jordan, 2003; López-Gatius, 2003; De Vries \& Risco, 2005). El número lactancia es también un factor conocido que afecta los indicadores reproductivos (Weigel, 2004; Winding et al., 2005). Otra fuente de variación en los indicadores reproductivos es el tipo de servicio utilizado (Williamson et al., 1978; De Vries et al., 2005; Olynk \& Wolf, 2008; Lima et al. 2009; Lima et al., 2010), no obstante existen pocos estudios que estudian la interacción de dicho factor con el nivel productivo a escala de rodeo lechero no experimental.

El objetivo de este trabajo fue estimar la contribución relativa en la determinación de la probabilidad de preñez a los 100 días de lactancia (DEL) de los siguientes factores: nivel productivo (NP), estación del parto (EP), número de lactancia (\# Lact), tipo de servicio (TS) (inseminación artificial —IA-, combinado o natural) y por último, la interacción del nivel productivo y el tipo de servicio.

\section{MATERIALES Y MÉTODOS}

\section{Base de datos}

Se trabajó con una base de datos de 162.116 lactancias pertenecientes a 291 tambos monitoreados durante dos años consecutivos (enero de 2007 a diciembre de 2008), fundamentalmente de la zona centro y sur de Santa Fe y provincia de Córdoba, ya que algunos tambos pertenecen a la provincia de Buenos Aires. Todos los tambos son usuarios del programa reproductivo Dairy Comp305 $\AA$. Se acondicionó la base de datos original de acuerdo a los objetivos de este trabajo, por lo que se descartaron las lactancias que tenían algún dato faltante de días en lactancia o para 305EM. Asimismo, se eliminaron las lactancias cuya duración era menor a 25 días y consecuentemente las lactancias en donde había una acumulación de litros menor a 125 a los 25 días de lactancia (25 días $\times 5$ litros/ día=125 litros). Luego, se clasificaron a las lactancias por el nivel productivo; para ello se utilizó la variable 305 equivalente maduro (305EM), la cual normaliza registros provenientes de distintas lactancias tanto en número como duración.

Para la clasificación de las lactancias se calculó el percentil 33 (P33) y 66 (P66) de la producción por lactancia para cada tambo; así lactancias de un mismo tambo con valores de 305EM menores al P33 conformaron el grupo de baja producción (BP), las lactancias con valores de 305EM mayores al P33 y menores al P66 conformaron el grupo de media producción (MP) y las lactancias con valores mayores al P66 conformaron el grupo de alta producción (AP). De esta manera se controló el efecto tambo mediante la agrupación de las lactancias marginales dentro cada tambo. Para clasificar el tipo de servicio, se lo consideró de IA cuando tenía el código de IA, natural cuando el código era de toro y combinado si había sido inseminado una a tres veces y luego se repasaba con el toro. Todos los servicios fueron aplicados posteriormente a la detección de celos. No se pudo controlar el periodo de espera voluntario, ya que cada tambo usa su propio criterio, y por esto se calculó el número de servicios dados hasta los 100 DEL y los DEL al primer servicio para cada nivel de producción, a fin de ver el comportamiento. Con respecto a la estación del parto, las lactancias que comenzaron entre los meses de diciembre a febrero se las clasificó como provenientes de partos de verano; las de marzo a mayo, de otoño; las de junio a agosto, de invierno; por último, a las lactancias comenzadas entre septiembre y noviembre se las agrupó en primavera.

\section{Análisis estadístico}

Inicialmente se realizó un análisis estadístico exploratorio de la variable TP acumulada a los 100 días de lactancia y 305EM para cada grupo de producción, en función de cuatro factores: nivel productivo (alta, media y bajo), estación de parto (verano, otoño, invierno y primavera), número de lactancia $(1,2,3$ y $\geq 4)$ y tipo de servicio utilizado (natural, combinada e IA). También, se midió el número de servicios dados hasta los 100 DEL promedios y los DEL promedio al primer servicio para cada nivel productivo. Se obtuvieron curvas de sobrevida para comparar a través de los DEL la TP acumulada. Las curvas se calcularon según el algoritmo de Kaplan y Meier para cada una de las categorías de la variable 305EM, y se comparó la igualdad de dos o más curvas de sobrevida con el estadístico Log Rank (Kaplan \& Meier, 1958). Un valor alto de Log Rank se corresponde a un valor 
"p" (probabilidad de que las curvas sean diferentes sólo por azar) pequeño; valores $p<0,05$ fueron usados como indicadores de diferencias estadísticamente significativas entre las curvas de sobrevida que en este trabajo sugieren la probabilidad de que un animal siga sin preñarse condicional al tiempo transcurrido desde el último parto hasta los 100 días de lactancia. Por último, se ajustó una regresión logística múltiple con el software JMP® versión 9.0.1 (JMP®, 2010). En el modelo se incluyeron como variables independientes el nivel productivo, la estación del parto, el número de lactancia al que pertenecía la lactancia bajo estudio y el tipo de servicio utilizado. Como salida de este modelo se obtuvieron los coeficientes de regresión y las razones de chances (Odds Ratios) que indican cómo las variables independientes introducen cambios en la probabilidad de la ocurrencia de un suceso, la preñez.

\section{RESULTADOS Y DISCUSIÓN}

\section{Análisis estadístico exploratorio}

En la Figura 1 se aprecia que los valores de la TP acumulada a los 100 DEL son mayores en las lactancias con un nivel productivo menor. El valor promedio de 305EM para las lactancias de alta fue de 9050 litros/lactancia, 7311 litros/lactancia para las de media y por último, 5473 litros/lactancia para las lactancias clasificadas como de baja producción (Tabla 1). El promedio de DEL al primer servicio y el promedio de número de servicios da-

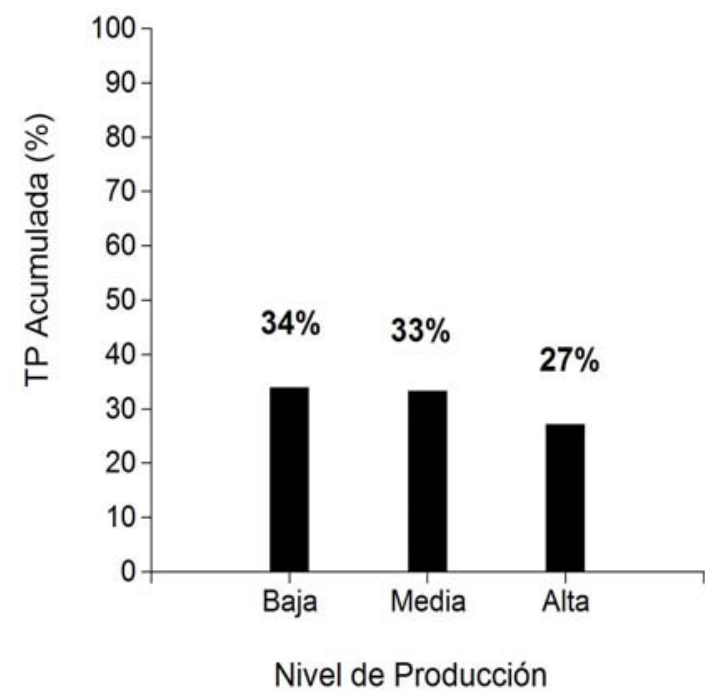

Figura 1. Distribución de la tasa de preñez (TP) acumulada a los 100 días en lactancia en función del nivel de producción.
Tabla 1. Media \pm E.E. de la variable producción de leche $305 E M^{1}$ (litros/lactancia) para cada factor: nivel de producción, estación de parto, número de lactancia y tipo de servicio aplicado.

\begin{tabular}{|c|c|c|c|}
\hline Factor & $\mathrm{n}$ & $\begin{array}{c}\text { Media } \\
\text { (litros/ } \\
\text { lactancia) }\end{array}$ & E.E. \\
\hline \multicolumn{4}{|l|}{$\begin{array}{l}\text { Nivel de } \\
\text { producción (NP) }\end{array}$} \\
\hline Baja & 53.754 & $5.473,40$ & 6,30 \\
\hline Media & 54.855 & $7.311,20$ & 6,30 \\
\hline $\begin{array}{l}\text { Alta } \\
\text { Estación del }\end{array}$ & 53.507 & $9.050,40$ & 8,80 \\
\hline $\begin{array}{l}\text { parto (EP) } \\
\text { Verano }\end{array}$ & 28.728 & $6.969,30$ & 12,80 \\
\hline Otoño & 51.361 & $7.263,90$ & 9,70 \\
\hline Invierno & 48.898 & $7.587,50$ & 10,40 \\
\hline $\begin{array}{l}\text { Primavera } \\
\text { Número de la } \\
\text { lactancia (\# Lact) }\end{array}$ & 33.129 & $7.100,10$ & 11,90 \\
\hline 1era & 49.450 & $7.483,50$ & 10,50 \\
\hline $2 \mathrm{da}$ & 39.747 & $7.464,50$ & 11,20 \\
\hline 3era & 29.453 & $7.280,00$ & 12,50 \\
\hline $\begin{array}{l}4 \text { ta ó > } \\
\text { Tipo de servicio } \\
\text { (TS) }\end{array}$ & 43.466 & $6.864,20$ & 10,00 \\
\hline Natural & 7.304 & $5.460,30$ & 17,00 \\
\hline Combinado & 18.279 & $5.798,90$ & 12,90 \\
\hline Inseminación artificial & 136.533 & $7.570,70$ & 5,90 \\
\hline
\end{tabular}

Tabla 2. Media \pm E.E. de la variable número de servicios dados hasta los 100 DEL (NServ.) y días a primer servicio (D1S) para cada nivel de producción.

\begin{tabular}{lcc}
\hline Factor & NServ. & D1S \\
\hline Nivel de producción (NP) & & \\
Baja & $1,43 \pm 0,00$ & $60,40 \pm 0,10$ \\
Media & $1,44 \pm 0.00$ & $62,30 \pm 0,09$ \\
Alta & $1,46 \pm 0.00$ & $63,60 \pm 0,09$ \\
\hline
\end{tabular}

dos hasta los 100 DEL para las lactancias de alta producción fue de 63 DEL y 1,46 servicios, 62 DEL y 1,44 servicios para las de media producción y por último 60 DEL y 1,43 servicios para las lactancias de baja producción (Tabla 2). En la Figura 2 se puede observar que los valores de la TP acumulada a los 100 DEL son mayores en las estaciones frescas que en las estaciones calurosas, y presenta los valores más altos de la TP acumulada la estación de invierno. En cuanto a los valores promedios de 305EM también fueron mayores en la estación de invierno (Tabla 1). Respecto al factor número de lactancia, la mayor TP acumulada correspondió a la primera y segunda lactancia, disminuyendo el porcentaje a medida que aumenta el número de lactancia (Figura 3). La misma tendencia se repi- 


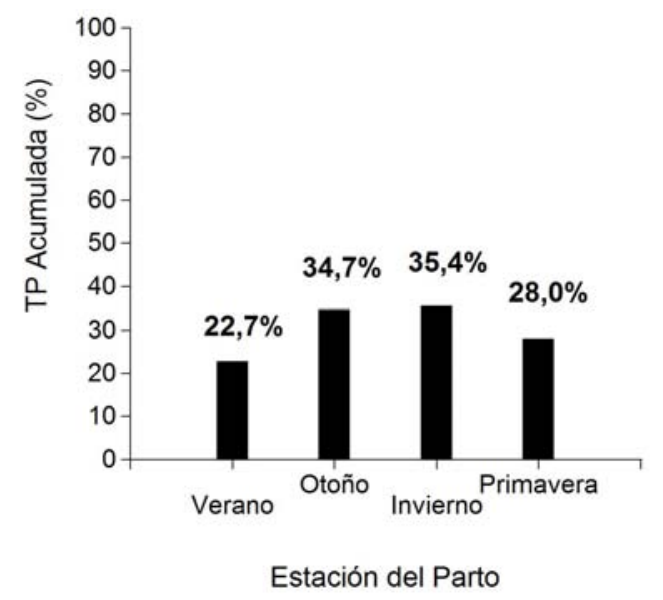

Figura 2. Distribución de la tasa de preñez (TP) acumulada a los 100 días en lactancia en función de la estación del parto.

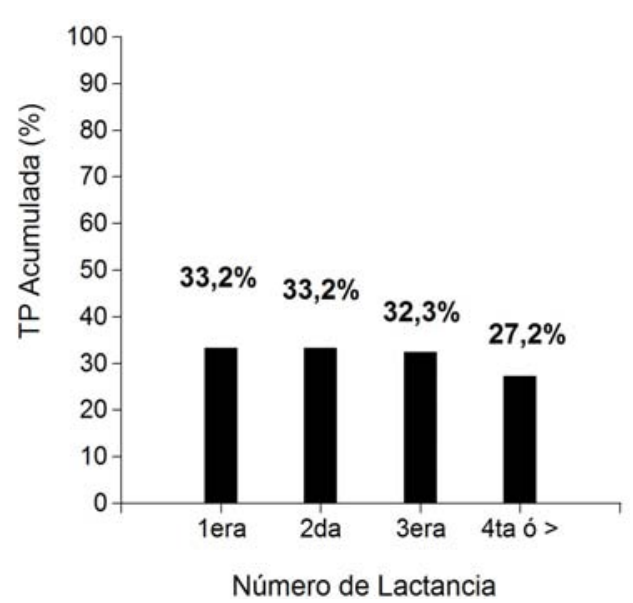

Figura 3. Distribución de la tasa de preñez (TP) acumulada a los 100 días en lactancia en función del número de lactancia.

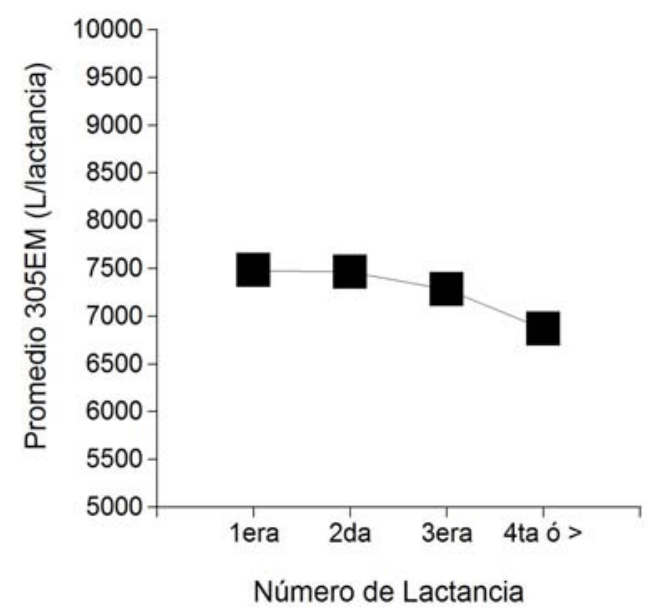

Figura 4. Gráfico de puntos de los valores promedios (L/lactancia) de la producción estimada que se espera alcance la vaca al ser adulta en 305 días de lactancia (305 equivalente maduro; 305EM) para cada número de lactancia.

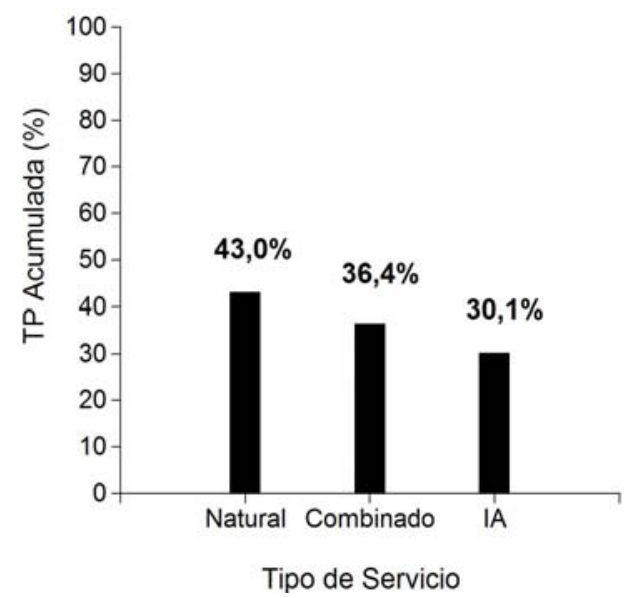

Figura 5. Distribución de la tasa de preñez (TP) acumulada a los 100 días en lactancia en función del tipo de servicio.

tió con la variable 305EM (Figura 4) (Tabla 1). Por último, en la Figura 5 se presenta la TP acumulada según el tipo de servicio aplicado en donde se aprecia que la mayor TP acumulada es cuando se utiliza como tipo de servicio al servicio natural. Sin embargo, aquí cabe destacar que los promedios del 305EM asociados con cada tipo de servicio utilizado difieren de manera considerable entre sí, con un promedio de 305EM para la natural de 5460 litros/lactancia y 7571 litros/lactancia para la IA (Tabla 1). En la Figura 6 se observa que para todos los niveles productivos con el servicio natural se obtuvieron los mayores valores de TP acumuladas a los 100 DEL. Así también, los resultados sugieren que la diferencia en porcentaje de la TP entre los niveles productivos es mucho mayor dentro del tipo de servicio natural que los alcanzados con la IA.

\section{Análisis de sobrevida}

En la Figura 7 se muestran las curvas de sobreviva construidas según particiones de las lactancias dadas por el nivel de producción de leche. Se observa nuevamente que las lactancias de alta producción se asocian a valores menores de preñez acumulada. A los 100 días desde el parto, las lactancias de alta producción se correspondieron con un $27 \%$ de animales preñados, mientras que las lactancias de baja producción se correspondieron con un 33\% de animales preñados. Las tres curvas de sobrevida, que indican el porcentaje de animales que se van preñando en función del tiempo transcurrido desde el inicio de la lactancia hasta los $100 \mathrm{DEL}$, mostraron diferencias estadísticamente significativas ( $p<0,0001)$. Tal vez estos resultados se encuentran sesgados por el efecto del periodo de espera voluntario, que como se aclaró 


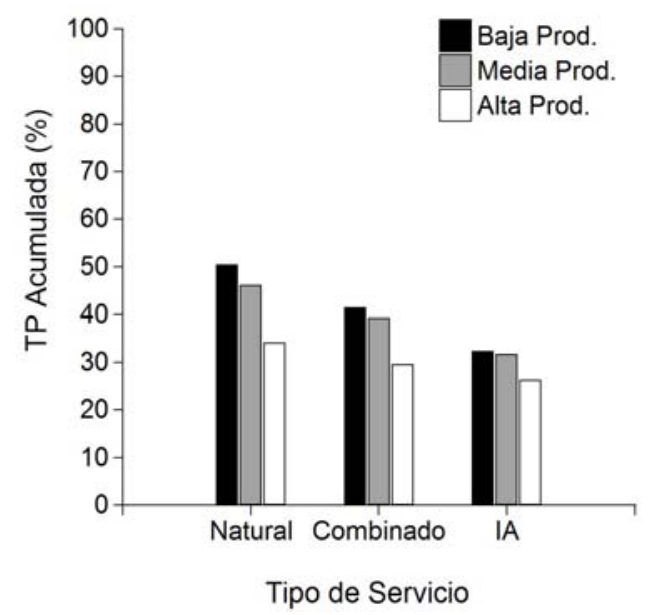

Figura 6. Distribución de la tasa de preñez (TP) acumulada a los 100 días en lactancia en función del tipo de servicio y nivel de producción.

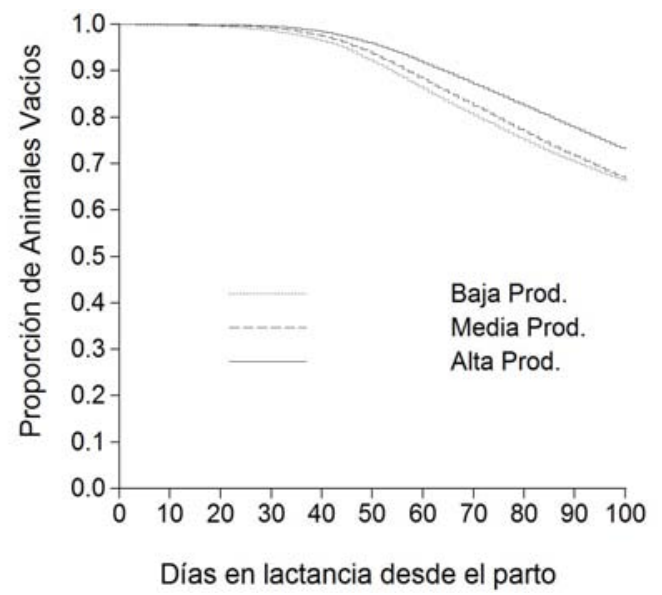

Figura 7. Curva de sobrevida de Kaplan-Meier. Días en lactancia que transcurren hasta lograr la preñez para lactancias con distintos niveles productivos. Estadístico Chi-cuadrado para media vs. baja: 13.275 ( $p=0,0002)$; estadístico chi-cuadrado para media vs. alta: $539,0(p<0,0001)$ y estadístico chi-cuadrado para baja vs. alta: $702.080(p<0,0001)$.

no se pudo controlar en este estudio, pero por ello se calculó el número de servicios (oportunidades) promedio que tuvieron hasta los $100 \mathrm{DEL}$ y los DEL promedio en que se realizó el primer servicio para cada nivel productivo. Si bien hay una diferencia de 3 días en los días al primer servicio entre las lactancias de alta y baja producción, el número de oportunidades promedio dados hasta los 100 DEL fue mayor en las lactancias de alta producción (1,46 veces versus 1,43 veces) (Tabla 2). En la Figura 8 se aprecian las curvas de sobrevida resultantes de la interacción nivel productivo y tipo de servicio utilizado, en donde se evidencia con claridad que las diferencias de la TP acumulada entre los distintos niveles productivos son menores cuando se utilizó IA como técnica (b) que cuando se utilizó servicio natural (a).

\section{Regresión logística}

Todas los factores propuestos en el modelo resultaron estadísticamente significativos $(P<$ 0,0001) (Tabla 3).

Tabla 3. Likelihood Ratio Test para cada factor analizado en la regresión logística múltiple.

\begin{tabular}{lrr}
\hline Factor & L-R ChiSquare & P $>$ ChiSq \\
\hline Nivel de producción & 556,20 & $<0,0001$ \\
Estación de parto & $2.151,80$ & $<0,0001$ \\
Número de lactancia & 625,50 & $<0,0001$ \\
Tipo de servicio & $1.004,30$ & $<0,0001$ \\
Interacción NP*TS & 45,20 & $<0,0001$ \\
\hline
\end{tabular}

Con respecto al nivel productivo, los resultados sugieren que afecta la probabilidad de la ocurrencia de la preñez (Tabla 4). Los valores de las razones de chances indican que las vacas con un nivel productivo bajo tienen mayores chances de preñarse antes de los $100 \mathrm{DEL}$ con respecto al nivel productivo tanto medio como alto, con valores de 1,12 y 1,71 respectivamente (Tabla 5). La alta producción de leche fue asociada con una concentración baja de estrógenos en plasma debido a su alta metabolización en el hígado (Lopez et al, 2004; Wiltbank et al, 2006). Por otra parte, numerosos estudios han asociado la disminución en la fertilidad de las vacas en los tambos con el aumento significativo de la producción de leche en los últimos 50 años (Dematawewa et al., 1998; Butler, 2000; Pryce \& Harris, 2004). Estos resultados no coinciden con los reportados por Bello et al. (2010), quienes estudiaron la relación entre la producción de leche y el desempeño reproductivo en vacas de primera parición de Michigan (EEUU), y concluyeron que la relación es heterogénea a través de los tambos, ya que en algunos no se encontró una relación antagónica, sino, por el contrario, sinérgica. Le Blanc (2010) ha sugerido que otros factores de manejo, como el nutricional y las condiciones de confort, deben tomarse en consideración y sostiene que si se encuentran cubiertos, no debería existir una relación antagónica entre la producción de leche y el desempeño reproductivo.

Los resultados sugieren que la estación en que se produce el parto, por lo tanto el comienzo de una nueva lactancia, afecta a la probabilidad de la ocurrencia de la preñez (Tabla 4). Las razones de 
Tabla 4. Parámetros estimados de la regresión para las variables explicativas del modelo de regresión logística múltiple.

\begin{tabular}{|c|c|c|c|c|}
\hline $\begin{array}{l}\text { Variable explicativa } \\
\end{array}$ & Coeficiente de regresión & Error Estándar & Chi-cuadrado & $\mathrm{P}>\mathrm{Chi}^{2}$ \\
\hline \multicolumn{5}{|l|}{ Nivel de producción (NP) } \\
\hline Produccion[Media] & 0,10 & 0,01 & 54,52 & $<0,0001^{*}$ \\
\hline Produccion[Alta] & $-0,32$ & 0,01 & 527,50 & $<0,0001$ \\
\hline \multicolumn{5}{|l|}{ Estación del parto (EP) } \\
\hline EP [Otoño] & 0,23 & 0,01 & 701,19 & $<0,0001$ \\
\hline EP[Invierno] & 0,28 & 0,01 & 992,37 & $<0,0001$ \\
\hline EP[Primavera] & $-0,12$ & 0,01 & 130,29 & $<0,0001$ \\
\hline \multicolumn{5}{|c|}{ Número de la lactancia (\# Lact) } \\
\hline \#Lact [1era] & 0,07 & 0,01 & 70,50 & $<0,0001$ \\
\hline \#Lact [2da] & 0,10 & 0,01 & 116,28 & $<0,0001$ \\
\hline \#Lact [3era] & 0,06 & 0,01 & 28,94 & $<0,0001$ \\
\hline \multicolumn{5}{|l|}{ Tipo de Servicio (TS) } \\
\hline TS [Combinado] & 0,00 & 0,01 & 0,10 & 0,7533 \\
\hline $\mathrm{TS}[\mathrm{IA}]$ & $-0,33$ & 0,01 & $1.028,02$ & $<0,0001$ \\
\hline \multicolumn{5}{|l|}{ Interacción (NP*TS) } \\
\hline [Combinado]*[Alta] & $-0,01$ & 0,02 & 0,33 & 0,5646 \\
\hline [Combinado] ${ }^{*}[$ Media] & 0,01 & 0,02 & 0,28 & 0,5949 \\
\hline$[\mathrm{IA}]^{*}[$ Alta $]$ & 0,09 & 0,01 & 41,92 & $<0,0001$ \\
\hline$[\mathrm{IA}]^{*}[$ Media $]$ & $-0,02$ & 0,01 & 2,81 & 0,0939 \\
\hline
\end{tabular}

*Valores $p<0,05$ indican diferencias estadísticamente significativas entre la clase especificada y la clase de referencia para el factor en estudio.

${ }^{1}$ La variable explicativa que no tiene valores de los parámetros estimados, es la referencia para las comparaciones estadísticas.

chances fueron: otoño versus primavera: 1,42 veces mayor; otoño versus verano: 1,87 veces mayor; invierno versus primavera: 1,49; invierno versus verano: 1,96 (Tabla 5) (Melendez \& Pinedo, 2007; Huang et al., 2009). Capitaine Funes et al. (2004) demostraron que en la estación de otoño e invierno los animales tienen mayor fertilidad que en las estaciones de primavera y verano. Estos resultados son de esperarse, ya que las vacas que paren en estas estaciones pasan la mayor parte o la totalidad de sus primeros 100 DEL en estas mismas estaciones, por lo tanto, se esperaría un mayor valor de TP acumulada si paren en las estaciones más frías. Además, los animales que paren en situaciones de stress calórico se ven afectados y se refleja en el resultado de los servicios al tacto de confirmación (Brouk et al., 2007; Morton et al., 2007).

Los coeficientes para la variable número de lactancias en el que se encuentra el bovino fueron estadísticamente significativos ( $p<0,001)$, con una tendencia cuadrática (Tabla 4). Según lo observado, las vacas de segunda lactancia son las que tienen mayor probabilidad de lograr la preñez a los 100 DEL. Las chances de preñez a los 100 DEL de los bovinos que se encuentran en su primera lactancia fueron de 0,97, 1,02 y 1,36 veces con respecto a los bovinos de la segunda, tercera y cuarta lactancia respectivamente. Estos resultados aseveran la hipótesis de la mayor fertilidad que po- seen los animales más jóvenes con respecto a vacas más viejas (Weigel, 2004; Windig et al., 2005; Melendez et al., 2007; Santos et al., 2009).

Con respecto al tipo de servicio utilizado y cen-

Tabla 5. Razón de chances (OR) e intervalo de confianza al 95\% (LI: límite Inferior y LS: límite superior) para comparaciones entre las clases de nivel productivo (NP), estación del parto (EP), número de lactancia (\#Lact) y tipo se servicio (TS) para especificar su efecto sobre tasa de preñez (TP) acumulada a los 100 días en lactancia.

\begin{tabular}{lccccc}
\hline Factores $^{1}$ & OR & LI 95\% LS 95\% & P>Chi \\
\hline NP [Baja vs. Alta] & 1,71 & 1,63 & 1,79 & $<0,0001^{*}$ \\
NP [Baja vs. Media] & 1,12 & 1,07 & 1,18 & $<0,0001$ \\
EP [Otoño vs. Primavera] & 1,42 & 1,37 & 1,46 & $<0,0001$ \\
EP [Otoño vs. Verano] & 1,87 & 1,80 & 1,93 & $<0,0001$ \\
EP [Invierno vs. Primavera] & 1,49 & 1,44 & 1,53 & $<0,0001$ \\
EP [Invierno vs. Verano] & 1,96 & 1,89 & 2,03 & $<0,0001$ \\
\#Lact. [1era vs. 2da] & 0,97 & 0,94 & 1,00 & 0,0574 \\
\#Lact. [1era vs. 3era] & 1,01 & 0,98 & 1,05 & 0,2680 \\
\#Lact. [1era vs. 4ta ó $>$ ] & 1,36 & 1,32 & 1,39 & $<0,0001$ \\
TS [Natural vs. IA] & 1,95 & 1,85 & 2,04 & $<0,0001$ \\
\hline
\end{tabular}

*Valores $\mathrm{p}<0,05$ indican diferencias estadísticamente significativas entre la clase especificada y la clase de referencia para el factor en estudio.

${ }^{1}$ La variable explicativa que no tiene valores de los parámetros estimados, es la referencia para las comparaciones estadísticas. 
trándose en la comparación entre la IA y el servicio natural, los resultados sugieren que las vacas que recibieron servicio natural tuvieron mayor chance de preñarse que las que recibieron IA o servicio combinado con un OR de 1,94 y 1,38, respectivamente (Tabla 5). Sin embargo, cuando se particionó el análisis según los niveles productivos de las lactancias se encontró que, como ya observó en la Figura 8, la diferencia en la TP acumulada es menor con el uso de IA que con el servicio natural a los 100 DEL entre los distintos niveles productivos. Varios trabajos han reportado sobre la conveniencia de la utilización de IA a tiempo fijo y otras técnicas para situaciones de alta producción de leche en donde la detección del celo se dificulta (De Vries et al., 2005; Olynk \& Wolf, 2008; Lima et al. 2009; Lima et al., 2010).
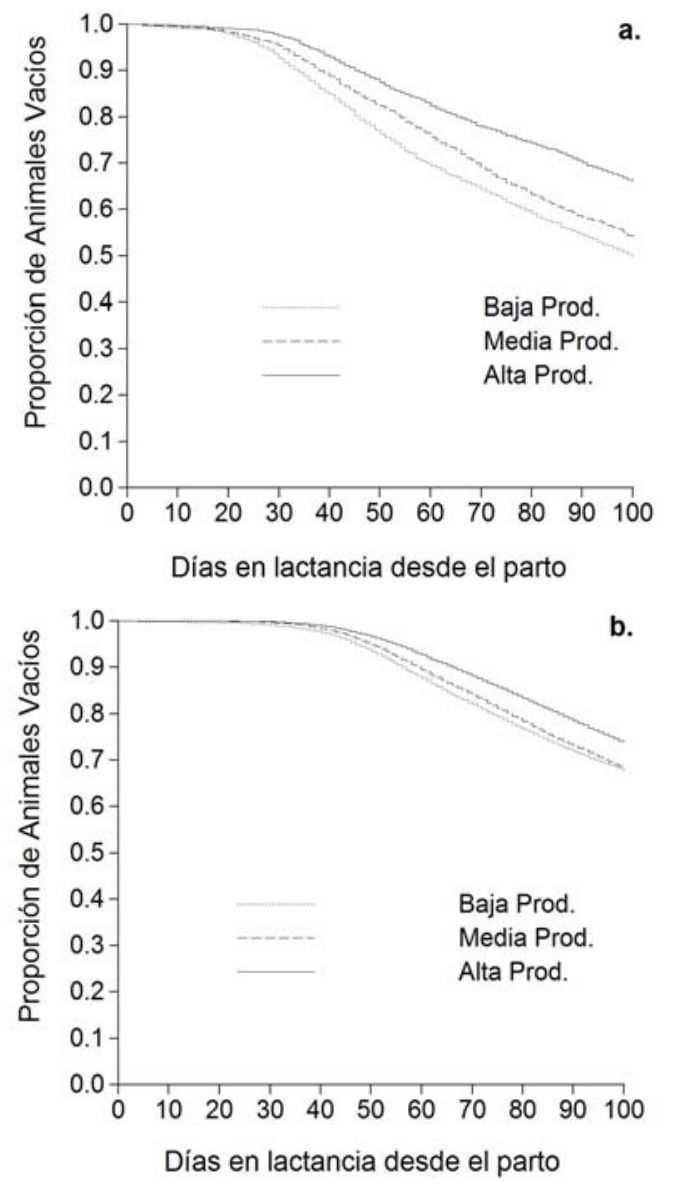

Figura 8. Curvas de sobrevida de Kaplan-Meier. Días en lactancia que transcurren hasta lograr la preñez para lactancias con distintos niveles productivos dentro del tipo de servicio natural (a) (estadístico chi-cuadrado $=152.890 ; p<0,0001$ ) e $I A$ (b) (estadístico chi-cuadrado= 535.720; $\mathrm{p}<0,0001$ ).

Es necesario notar en este trabajo que al servi- cio natural generalmente se lo asocia con niveles productivos bajos, y la IA con niveles productivos altos, como se detectó en la exploración de los datos de controles lecheros de este estudio (Tabla 1) (Capitaine Funes, 2002; Capitaine Funes et al., 2003). Los resultados sugieren la necesidad de combinar datos productivos y reproductivos para determinar la eficiencia económica de un animal. Además, el impacto del mes del parto es un factor importante para diseñar modelos productivos que aumenten la rentabilidad de manera sustentable.

\section{CONCLUSIONES}

La tasa de preñez acumulada a los 100 días en lactancia se ve afectada por: el nivel productivo, a mayor producción menores son la chances para preñarse a los 100 DEL; la estación de parto, a estaciones más frías mayores son las chances de preñarse a los 100 DEL; el número de lactancia, la relación resultó cuadrática; el tipo de servicio empleado, las chances de preñarse a los 100 DEL utilizando servicio natural son mayores; y por último, la interacción entre el nivel productivo y tipo de servicio, en donde hay que destacar que aunque con IA no se detectó mayor tasa de preñez acumulada a los 100 días en lactancia, respecto al servicio natural, se observó que la IA estuvo asociada a niveles productivos mayores para todos los grupos marginales de producción (baja, media y alta).

Por lo tanto, los resultados sugieren la necesidad de diseños alternativos y evaluación del manejo reproductivo según niveles productivos específicos como herramienta para incrementar la eficiencia reproductiva global y la rentabilidad del sistema.

\section{BIBLIOGRAFÍA}

Bello, N.M.; J.P. Steibel and R.J. Tempelman, 2010. Hierarchical Bayesian modeling of radom and residual variance-covariance matrices in bivariate mixed effects models. Biometrical Journal 52(3): 297-313.

Butler, W.R. 2000. Nutritional interactions with reproductive performance in dairy cattle. Animal Reproduction Science 60:449-457.

Brouk, M.J.; J.P. Harner; J.F. Smith and D.V. Armstrong, 2007. Environmental modifications to address heat stress. J. Dairy Sci. 90(Suppl. 1):624. (Abstr.)

Capitaine Funes, A. 2002. Monitoreo de la eficacia reproductiva de rodeos lecheros. Primeras jornadas Taurus de Reproducción Bovina. Buenos Aires, Argentina.

Capitaine Funes, A. 2005. Factores q afectan la tasa de preñez en rodeos lecheros en Argentina. 2005. VI Sim- 
posio internacional de reproducción animal. Pag.179196.

Capitaine Funes, A.; A. Vater y N. Acosta, 2003. Análisis reproductivo de rodeos lecheros usuarios del Dairy Comp305. Revista Taurus Año 5 No17 abril 2003. 1428.

Capitaine Funes, A.; M.I. Oyarzabal; G.A. Bo and A. Vater, 2004. Factors affecting conception rate in Argentinian dairy herds. 15 th International Congress on Animal Reproduction, 2004, Abstract Volume 2. Page 289.

De Vries, A. and C.A. Risco, 2005. Trends and seasonality of reproductive performance in Florida and Georgia dairy herds from 1976 to 2002. J. Dairy Sci. 88:31553165.

De Vries, A.; C. Steenholdt and C.A Risco, 2005. Pregnancy rates and milk production in natural service and artificial indeminated dairy herds in florida and Georgia. J.Dairy Sci. 88: 948-956.

Dematawewa, C.M.B. and P.J. Berger, 1998. Genetic and phenotypic parameters for 305-day yield, fertility, and survival in Holsteins. J. Dairy Sci. 81:2700-2709.

Ferguson, S.D. and D.T. Galligan, 1993. Reproductive programs in dairy herds. Proc. Centr. Vet. Conf. 161178.

Huang, C.; S. Tsuruta; J.K. Bertrand; I. Misztal; T.J. Lawlor and J.S. Clay, 2009. Trends for conception rate of Holstein over time in the southeastern United States. J. Dairy Sci. 92: 4641-4647.

Indicadores Lácteos. 2009. Convenio Lechería - S.A.G.P. y A.- C.I.L. - F.I.E.L; Elaboración: Lácteos - Dirección de Industria Alimentaria - S.A.G.P. y A Link: http://www. alimentosargentinos.gov.ar/lacteos/docs/02_Nacional/indica/Indica09_07.hm Consultado 15/01/2011.

JMP $®$ 9.0.1 Copyright@. 2010. SAS Institute Inc. Software for Windows. Cary, NC, USA.

Jordan, E.R. 2003. Effects of heat stress on reproduction. J.Dairy Sci. 86: (E. Suppl.): E104-E114.

Kaplan, E.L. and P. Meier, 1958. Nonparametric estimation from incomplete observations. J. Amer. Statist. Assn. 53:457-481.

Leblanc, S. 2010. Assesing the association of the level of milk production with reproductive performance in dairy cattle. J.Reprod Dev S1-S7.

Lima F.S.; C.A. Risco; M.J. Thatcher; M.E. Benzaquen; L.F. Archbald; J.E.P. Santos and W.W. Thatcher, 2009. Comparison of reproductive performance in lactating cows bred by natural service or timed artificial insemi- nation. J. Dairy Sci. 92:5456-5466.

Lima F.S.; A. De Vries; C.A. Risco; J.E.P. Santos and W.W. Thatcher, 2010. Economic comparison of natural service and timed artificial insemination breeding programs in dairy cattle. J.Dairy Sci. 93: 4404-4413.

Lopez, H.; L.D. Satter and M. Wiltbank, 2004. Relationship between level of milk production and estrous behaviour of lactating dairy cows. Animal Reproductive Science, 81: 209-223.

López-Gatius, F., 2003. Is fertility declining in dairy cattle? A retrospective study in northeastern Spain. Theriogenology 60:89-99.

Lucy, M. C. 2001. Reproductive loss in high-producing dairy cattle: Where will it end? J. Dairy Sci. 84:12771293.

Melendez, P. and P. Pinedo, 2007. The Association between reproductive performance and milk yield in Chilean Holstein cattle. J. Dairy Sci. 90:184-192.

Morton, J.M.; W.P. Tranter; D.G. Mayer and N.N. Jonsson, 2007. Effects of environmental heat on conception rates in lactating dairy cows: Critical periods of exposure. J. Dairy Sci. 90:2271-2278.

Olynk, N.J. and C.A. Wolf, 2008. Economic Analysis of reproductive management strategies on US commercial dairy farms. J. Dairy Sci. 91: 4082-4091.

Pryce, J.E. and B.L. Harris. 2004. Genetic and economic evaluation of dairy cow body condition score in New Zealand. Interbull Bull. 32:82-85.

Santos, J.E.P; H.M. Rutigliano and M.F. Sá Filho, 2009. Risk factors for resumption of postpartum cyclicity and embryonic survival in lactating dairy cows. Anim. Reprod. Sci. 110:207-221.

Weigel, K.A, 2004. Improving the reproductive efficiency of dairy cattle through genetic selection. J. Dairy Sci. 87(E Suppl.): E86-E92.

Williamson, N.B; R.S. Morris and G.A. Anderson, 1978. Pregnancy rates and non return rates following artificial and natural breeding in dairy herds. Aust. Vet. J. 54:111-120.

Wiltbank, M.; H. Lopez; R. Sartori; S. Sangsritavong and A. Gümen, 2006. Changes in reproductive physiology of lactating dairy cows due to elevated steroid metabolism. Theriogenology 65:17-29.

Windig, J.J.; M.P.L Calus and R.F. Veerkamp, 2005. Influence of herd environment on health and fertility and their relationship with milk production. J. Dairy Sci. 85:335-347. 